\title{
OPTIMIZATION OF SPAR MASS AND FUSELAGE LOCATION TO DECREASE LOSS OF AIRPLANE LIFT FORCE
}

\author{
A. Ohapkin*, S. Serokhvostov** \\ * Central Aerohydrodynamic Institute (TsAGI), Zhukovsky St., 1, Zhukovsky, 140180, Moscow Region, Russia \\ E-mail: alex@sky.falt.ru \\ ** Central Aerohydrodynamic Institute (TsAGI), Zhukovsky St., 1, Zhukovsky, 140180, Moscow Region, Russia \\ Moscow Institute of Physics and Technology (MIPT), Department of Aeromechanics and Flight Engineering (DAFE), \\ Gagarina St. 16, Zhukovsky, 140180, Moscow Region, Russia.E-mail: serokhvostov@aviel.ru \\ Received 1511 2006, accepted 10092007
}

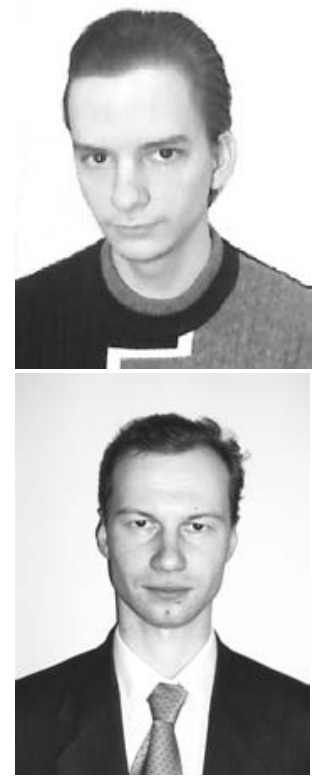

Alexey OHAPKIN, PhD Student

Date and place of birth: 1982, Ivanovo Region, Russia.,

Education: Master's degree, Moscow Institute of Physics and Technology (MIPT), 2006

Present position: Junior Researcher, PhD student (TsAGI).

\begin{abstract}
Considered in this paper is the problem of optimizing spar mass to minimize lift force loss for a set of spar cross-section shapes in cases of constant and elliptical lift force distributions. The main idea is that the deformation of the spar under aerodynamic and gravitational forces causes a decrease in lift force and that there must be some optimal spar strength that gives a minimum for the sum of the loss of lift force and spar weight. The influence of fuselage location on the loss of lift force in the case of multi-fuselage design is also investigated. The behaviour of lift loss as a function of the location of fuselages is discussed.
\end{abstract}

Keywords: HALE, spar mass, wing deformation, lift force loss.

\section{Introduction}

The wing of a real aircraft is deformed during flight due to non-uniform aerodynamic load and mass distribution. This effect results in loss of lift force.

Figure 1 shows the mechanism of this effect. One can see that the lift force of an arbitrary part of the wing is directed not vertically, but at some angle $\beta$ to the vertical direction.

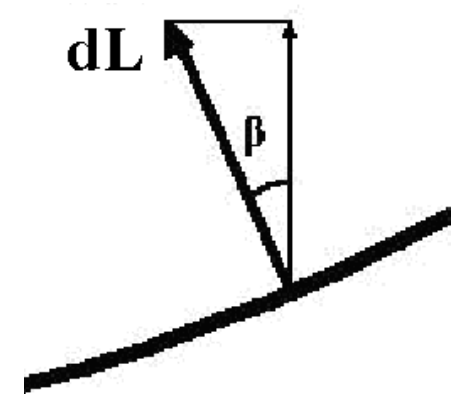

Fig 1. The direction of lift force with respect to the deformed wing 
The spar is the most important wing box structural component that defines the value of wing deformation and, as a consequence, loss in lift force. Making the spar stronger (for example, by increasing the cross-section shape size) can decrease wing deformation and associated loss in lift force. On the other hand, the mass of the spar increases with the increase in its strength. So there must be some an optimal strength that provides the minimum of "spar weight + lift force loss".

In most cases, the value of the loss in lift force is not high enough to take into consideration. But, for example, the design of HALE aircraft requires a wing with a very large wingspan and high aspect ratio to achieve desired aerodynamic performance. Wing deformation grows to a considerable amount with the increase in the wingspan and aspect ratio, and therefore deformations and related loss in lift force cannot be neglected (Fig 2). In such a special case, aerodynamic and structural weight optimization plays an important role in the aircraft design procedure.

The problem of maximizing the value of "lift force minus weight of aircraft" through the variation of spar mass is investigated.

The wing spar can be of various types. For example, the spar of "Helios" has constant cross-section shape and dimensions. This type of spar is preferable from a "manufacturing" point of view. But a spar with constant maximal mechanical tension can have less mass than a spar with a constant cross-section with the same deformation.

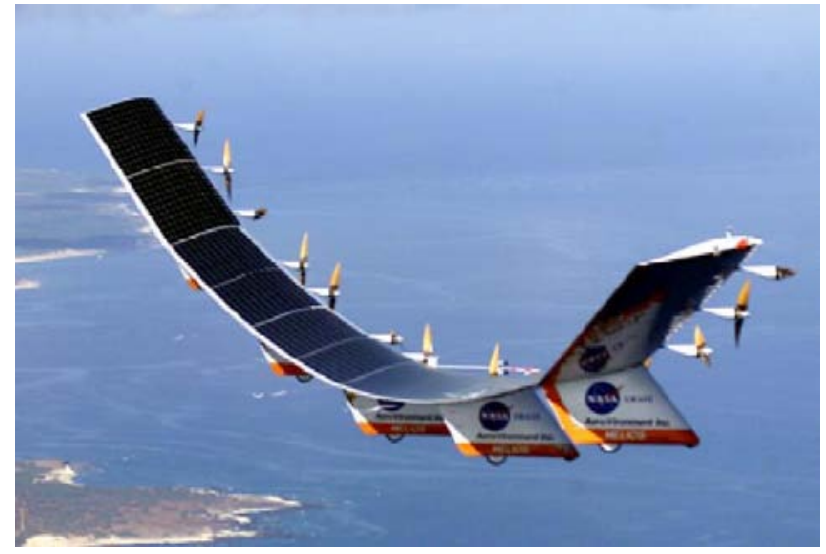

Fig 2. Wing deformation of HALE "Helios" [1]

There can also be various types of lift force distribution along the wing span. Elliptical distribution provides a minimum of induced drag. But it is rather difficult to realize this distribution. The other model case is constant lift distribution.

Figure 3 shows the distribution of lift force for "Helios". One can see that this distribution is more complex than the types mentioned above but can be approximated by these types of distribution in the first steps of the procedure of spar design.

Cases of constant cross-section spar and a spar with constant tension for constant and elliptical lift force distribution will be considered.

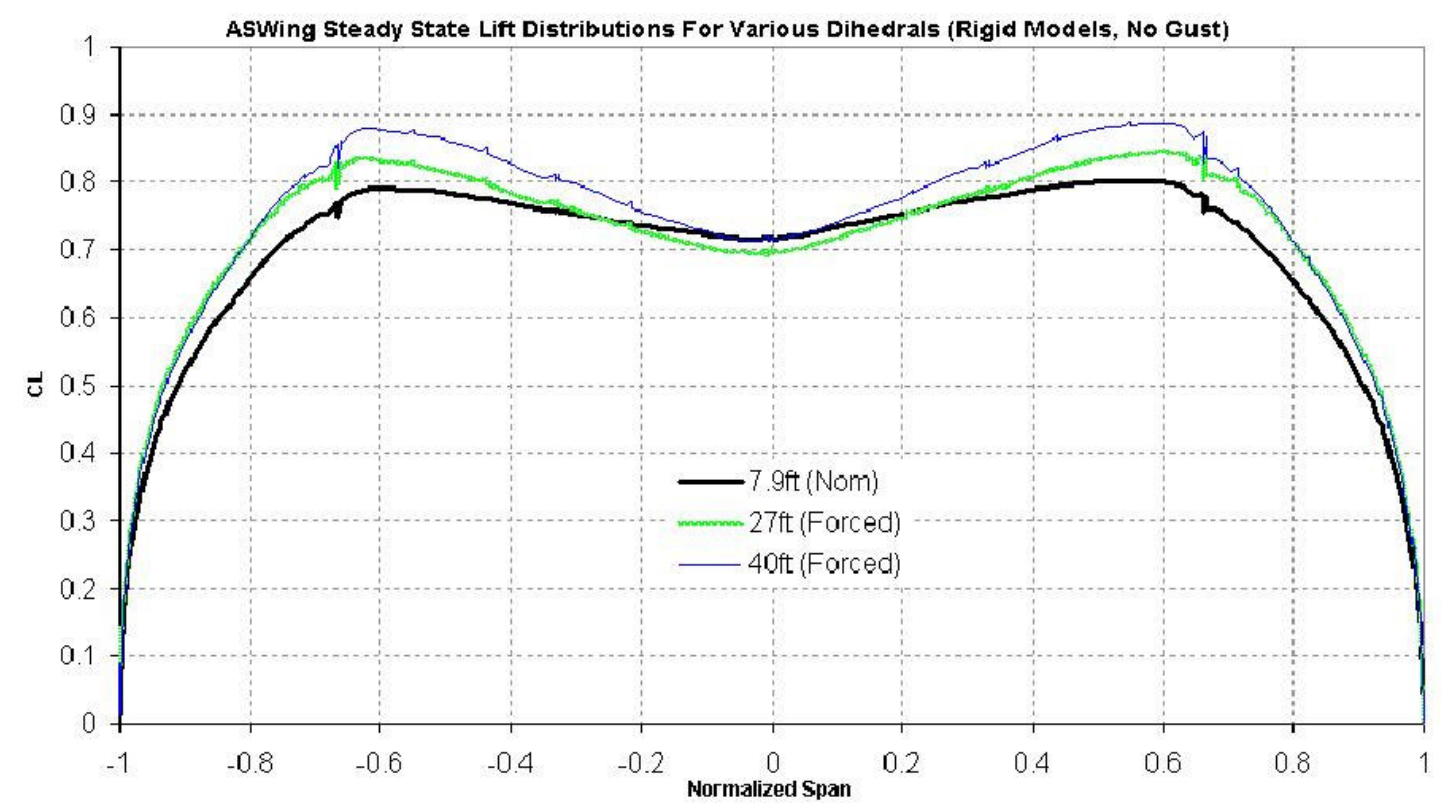

Fig 3. "Helios" lift force distribution [1]

\section{Spar mass optimization}

Consider the procedure of this investigation in the case of constant spar cross-section, constant wing chord $b$, and constant lift distribution.

The lift force $L^{*}$ of a deformed wing with span $b$ and chord $c$ can be obtained by the formula

$$
L^{*}=2 \int_{0}^{\frac{b}{2}} \frac{C_{L} \rho V^{2} c}{2} \cos \beta d \xi
$$

where

$$
\cos \beta=\frac{1}{\sqrt{1+\operatorname{tg}^{2} \beta}}=\frac{1}{\sqrt{1+z^{\prime 2}}}
$$


$C_{L}-$ lift force coefficient,

$\rho$ - air density,

$V$ - velocity of aircraft,

$z$ - wing chord vertical coordinate.

As we know from the theory of elasticity ${ }^{*}$,

$$
z^{\prime}=\int_{0}^{y} \frac{M_{x}}{E J} d z=\frac{C_{L} \rho V^{2} c}{12 E J}\left[y^{3}-\frac{3}{2} y^{2} b+\frac{3}{4} y b^{2}\right]
$$

where $M_{x}-$ mechanical torque,

$E$ - Young module,

$J$ - inertia moment of the cross-section,

$y$ - coordinate of the cross-section along the wing span.

From this, the lift force is defined by the formula

$$
L^{*}=\frac{C_{L} \rho V^{2} S}{2}\left[1-\frac{1}{28}\left(\frac{C_{L} \rho V^{2} S b^{2}}{2 E J}\right)^{2}\right]=L_{0}-A_{1} \frac{L_{0}{ }^{3}}{m_{l}{ }^{2}},
$$

where

$$
L_{0}=\frac{C_{L} \rho V^{2} S}{2}, A_{1}=\frac{b^{6} \rho_{l}^{2}}{448 E^{2}(\bar{c} c)^{4}},
$$

$S$ - wing area,

$l$-wing span,

$\rho_{l}$ - spar density,

$\bar{c}$ - relative chord thickness.

During cruise flight with constant velocity and altitude, lift force must be equal to the airplane weight:

$$
L^{*}=m g
$$

where $m$ is airplane mass.

The optimization problem is searching for the maximum of function $F$ :

$$
F=m-m_{l}
$$

at condition (1).

So, the Lagrange function $Q$ for this problem is

$$
Q=m-m_{l}+\lambda\left(L_{0}-A_{1} \frac{L_{0}{ }^{3}}{m_{l}{ }^{2}}-m g\right) .
$$

From this, optimal spar mass is defined as

$$
m_{l}=\sqrt[3]{\frac{2 A_{1}}{g}} L_{0}
$$

and the minimum value of lift force relative losses $B$ is

$$
B=\frac{L_{0}-L^{*}}{L_{0}}=\sqrt[3]{\frac{A_{1} g^{2}}{4}}
$$

It must be noted that this spar mass can correspond to the case in which the mechanical tension in some spar points exceed the limits, so the spar may be damaged. It is rather evident that the highest tension value $\sigma_{\max }$ is in the wing root. Its value can be obtained from the theory of elasticity as

$$
\sigma_{\max }=\frac{Y_{0} b^{2} \rho_{l}}{4 \bar{c} c m_{l}}
$$

For the case considered

$$
\sigma_{\max }=\sqrt[3]{\frac{7}{2} g E^{2} \bar{c} c \rho_{l}}
$$

The investigation shows that for all cases mentioned above the expressions for $m_{l}, \sigma_{\max }$ and $B$ are the same and differs from each other only by the numerical constants $A_{0}$ and $D_{0}$ :

$$
\begin{gathered}
m_{l}=\sqrt[3]{\frac{2 A_{0} b^{6} \rho_{l}^{2}}{g E^{2}(\bar{c} c)^{4}} L_{0} \sim \sqrt[3]{A_{0}}} ; \\
B=\frac{L_{0}-L^{*}}{L_{0}}=\sqrt[3]{\frac{A_{0} b^{6} \rho_{l}^{2} g^{2}}{4 E^{2}(\bar{c} c)^{4}}} \sim \sqrt[3]{A_{0}} ; \\
\sigma_{\max }=\sqrt[3]{D_{0} g E^{2} \bar{c} c \rho_{l}} .
\end{gathered}
$$

So, the relationships between the values of these functions for all the cases considered are presented in table. From this table, one can see that for the constant values of wing span, wing chord, and characteristics of spar material, it is possible to reduce the relative lift force losses and maximal tension through rational lift and spar cross-section distribution along the wing span.

\section{Optimization of fuselage location}

Another way to decrease losses in lift force is multifuselage design and proper location of the fuselages.

Consider the problem of lift force maximization through the location of fuselage optimization for the cases of two, three, four and five fuselages. Assume that fuselages are located symmetrically with respect to the centre line of the airplane.

Let $\varepsilon$ be the relative mass of all fuselages with respect to total aircraft mass.

Consider the case of two fuselages. The fuselages are the point masses located at the distance $y_{0}$ from the centre line. So, from the theory of elasticity [2],

*) ПИСАРЕНКО, ГС., ЯКОВЛЕВ, АП., МАТВЕЕВ, ВВ. Справочник по сопротивлению материалов. Киев: Наукова думка, 1975. 
Table. Results obtained for the cases of spar cross-section and lift distribution investigated

\begin{tabular}{|l|c|c|c|c|}
\hline & $A_{0}$ & $m_{l}$ & $B$ & $\sigma_{\max }$ \\
\hline $\begin{array}{l}\text { Constant lift force distribution, constant spar } \\
\text { cross-section }\end{array}$ & $A_{00}$ & $\mathrm{~m}_{0}$ & $B_{0}$ & $\sigma_{0}$ \\
\hline $\begin{array}{l}\text { Constant lift force distribution, constant spar } \\
\text { tension }\end{array}$ & $A_{00} / 1.93$ & $\mathrm{~m}_{0} / 1.245$ & $B_{0} / 1.245$ & $\sigma_{0} / 2.41$ \\
\hline $\begin{array}{l}\text { Elliptical lift force distribution, constant spar } \\
\text { cross-section }\end{array}$ & $A_{00} / 1.9$ & $\mathrm{~m}_{0} / 1.238$ & $B_{0} / 1.238$ & $1.05 \sigma_{0}$ \\
\hline $\begin{array}{l}\text { Elliptical lift force distribution, constant spar } \\
\text { tension }\end{array}$ & $A_{00} / 4.57$ & $\mathrm{~m}_{0} / 1.66$ & $B_{0} / 1.66$ & $\sigma_{0} / 2.41$ \\
\hline
\end{tabular}

$$
\frac{L^{*}\left(y_{0}\right)}{C_{L} \rho V^{2} c}=\int_{0}^{y_{0}}\left[1-\frac{1}{2}\left(\int_{0}^{y} \frac{\varepsilon m g}{2 \delta}\left[\frac{1}{b}\left(\frac{b}{2}-\xi\right)^{2}-\left(y_{0}-\xi\right)\right] d \xi\right)^{2}\right] d y+\int_{y_{0}}^{\frac{b}{2}}\left[1-\frac{1}{2}\left(\int_{0}^{y} \frac{\varepsilon m g}{2 b l}\left[\frac{b}{2}-\xi\right]^{2} d \xi-\int_{0}^{y_{0}} \frac{\varepsilon m g}{2 \delta}\left[y_{0}-\xi\right] d \xi\right)^{2}\right] d y
$$

By varying the variable $y_{0}$, one can obtain the maximal value of $L^{*}$. For this case, optimum corresponds to

$$
y_{0}^{*}=0,56 \frac{b}{2} .
$$
figure 4.

The behavior of $L^{*}$ as a function of $y_{0}$ is shown in

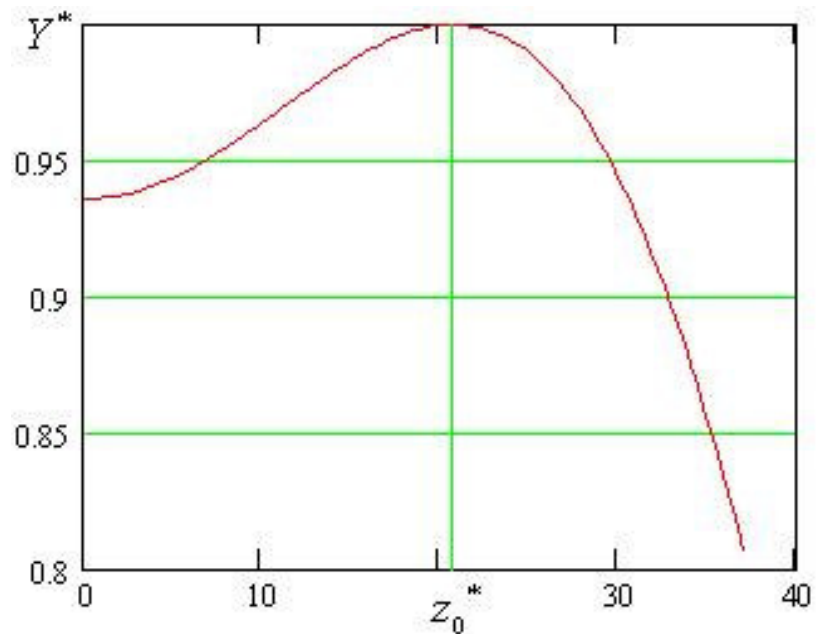

Fig 4. Lift force as function of fuselage location for the case of two fuselages

The same procedure for three fuselages gives

$$
y_{0}^{*}=0.7 \frac{b}{2} \text {. }
$$

In the case of four fuselages, $L^{*}$ is the function of two variables: $z_{0}$ and $z_{1}$ (locations of two fuselages along the semi-span). Calculation gives the optimum at

$$
y_{0}^{*}=0,24 \frac{b}{2}, y_{1}^{*}=0,78 \frac{b}{2} .
$$

For the case of five fuselages (as for "Helios") the reults of optimization are

$$
y_{0}^{*}=0.4 \frac{b}{2}, y_{1}^{*}=0.82 \frac{b}{2}
$$

It is also rather interesting to investigate the behaviour of function $L^{*}$ near the maximum. Figure 5 shows the isolines of this function. One can see that this function changes rather slowly in the vicinity of the maximum, so small displacement of fuselages cannot strongly affect the lift force. The same results can be obtained for the cases of two, three and four fuselages.

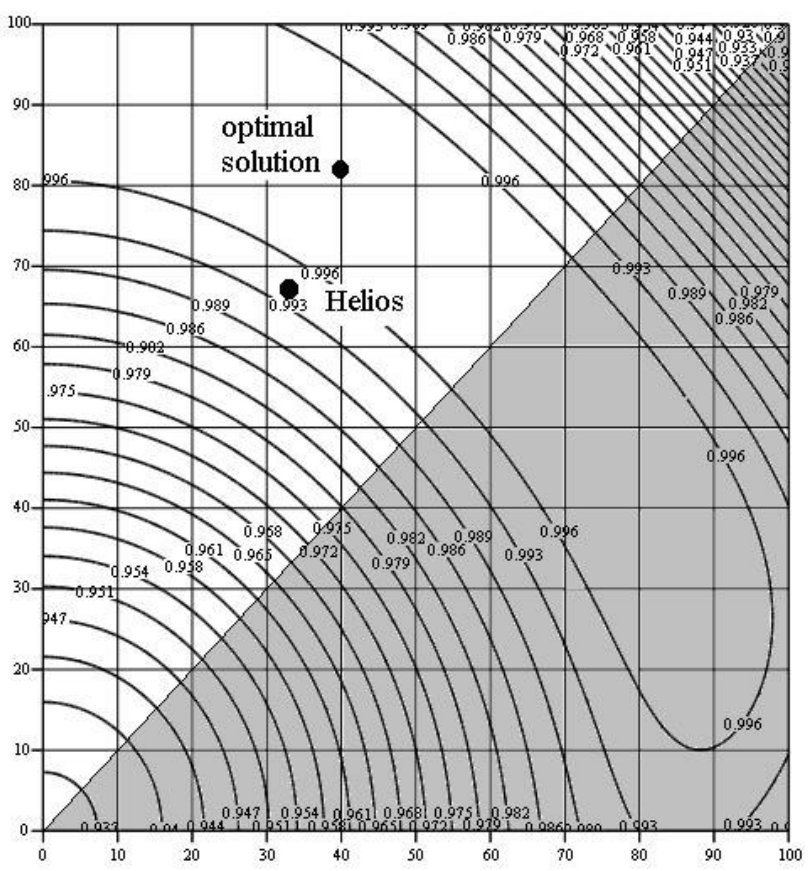

Fig 5. Isolines of lift force as function of fuselage location for the case of five fuselages 


\section{Conclusions}

1. The use of a spar with a rational value of strength can improve the characteristics of an airplane.

2. A spar with constant maximal tension and elliptical lift distribution can increase lift force and decrease spar mass and maximal value of tension.

3. Multi-fuselage design with properly located fuselages can increase lift force.

\section{References}

1. Investigation of the helios prototype aircraft mishap. Langlay Research Center, Jan 2004. 